\title{
Comparison of Reproductive Hormonal Profile and Sexual Performance Satisfaction among Type-2 Diabetes Mellitus and Controls
}

\author{
Sikandar Hayat Khan1', Roomana Anwar22, Zulfiqar Ali Amin ${ }^{3}$, Nadeem Fazal ${ }^{4}$, Athar Abbas Shah Gilani ${ }^{4}$ \\ and Muhammad Tariq ${ }^{5}$ \\ 1Department of Pathology, PNS Hafeez, Islamabad, Pakistan \\ ${ }^{2}$ Department of Biochemistry, Yusra Medical and Dental College, Islamabad, Pakistan \\ ${ }^{3}$ Department of Medicine, PNS Shifa, Islamabad, Pakistan \\ ${ }^{4}$ Department of Medicine, PNS Hafeez, Islamabad, Pakistan \\ ${ }^{5}$ Department of Healthcare Administration, PNS Hafeez, Islamabad, Pakistan
}

\begin{abstract}
Objective: To evaluate reproductive hormonal profile among three groups with varying sexual performance satisfaction (erectile dysfunction) with or without type-2 diabetes (T2DM).

Study Design: Comparative cross-sectional analysis.

Place and Duration of Study: Department of Pathology, PNS Hafeez Hospital, Islamabad, from January to December, 2018. Methodology: One hundred and twenty-one subjects including T2DM and age-matched controls were segregated into three groups based upon their sexual performance satisfaction. These groups were evaluated by one-way ANOVA for various anthropometric, glycemic indices and reproductive hormones and free androgen indices (FAl). A general linear model (GLM) was utilised using $\mathrm{HbA} 1 \mathrm{c}$ and FAl as dependent variable with sexual performance satisfaction as fixed and quantitative CRP and urinary albumin creatinine ratio (UACR) as random variables to evaluate diabetes complication and inflammation on sexual performance.

Results: Comparison between three groups suggested a rising trend for FAl as: FAl: \{Non-satisfied $(n=43): 41.78$ (95\%Cl:36.67-46.90)\}, \{Just satisfied $(n=38): 48.81(95 \% \mathrm{Cl}: 42.96-54.66)\}$, \{Satisfied $(n=40): 51.86(95 \% \mathrm{Cl}: 45.27-58.44)\}$, $[p=0.041]$. GLM model evaluation suggestion that for any particular degree of reported $E D, H b A 1 c$ demonstrated a higher trend from non-satisfied subjects to satisfied subjects with inflammation following a rise with $\mathrm{HbA} 1 \mathrm{c}$ levels, identifying inflammation as more related with worsening diabetes than with sexual performance satisfaction. FAl levels were higher among subjects who showed no erectile dysfunction than subjects with less satisfied groups with both inflammation (qCRP) and nephropathy (UACR) causing across the group decline for FAl among all ED groups.

Conclusion: Sexual performance satisfaction and FAI decline with rise in HbA1c. Moreover, subjects having nephropathy or higher inflammation (qCRP) were found to have lower FAI and ED, both in controls and T2DM.
\end{abstract}

Key Words: Type-2 diabetes mellitus (T2DM), Male hypogonadism, Testosterone, Sex hormone binding globulin (SHBG), Erectile dysfunction (ED), HbA1c.

How to cite this article: Khan SH, Anwar R, Amin ZA, Fazal N, Gilani AAS, Tariq M. Comparison of reproductive hormonal profile and sexual performance satisfaction among type-2 diabetes mellitus and controls. J Coll Physicians Surg Pak 2019; 29(12):1153-1158.

\section{INTRODUCTION}

Type-2 diabetes mellitus (T2DM) has major micro- and macro-vascular complications. ${ }^{1}$ Though macro-vascular complications like heart attacks and strokes are wellrecognised, appropriate considerations are now being given to nephropathy, neuropathy and retinopathy. ${ }^{2}$ However, due to societal norms about hypogonadism, these remain less clinically focused.3,4

Pathology of male hypogonadism remains multifactorial starting from ageing, anatomical disorders, genetic

Correspondence to: Dr. Sikandar Hayat Khan, Department of Pathology, PNS Hafeez, E-8, Islamabad, Pakistan

E-mail:sik_cpsp@yahoo.com

Received: January 22, 2019; Revised: September 21, 2019;

Accepted: September 23, 2019 diseases, injury, infections, certain tumors, medications, and other disorders. 5 In T2DM, the hypogonadism is probably resulting from hypogonadotropic hypogonadism as pathology is hypothalamic-pituitary-axis. However, studies have identified certain controversies in terms of hormonal disturbances in T2DM. Dandona et al. did not observe a link between low testosterone, HbA1c and diabetes duration. ${ }^{6}$ Similarly, $\mathrm{HbA} 1 \mathrm{c}$ was not found to be higher in T2DM with erectile dysfunction (ED) in a Turkish study. ${ }^{7}$ Lu et al. have demonstrated that $\mathrm{HbA} 1 \mathrm{c}$ was significantly related with ED in T2DM patients. ${ }^{8}$ Moreover, further contrasting data about hormonal profiles, diabetes markers and erectile dysfunction is found in literature.9,10

Hypogonadism within different societies are rarely discussed, poorly evaluated by physicians and results in 
large gaps in care provision. It is also important to consider the underlying hormonal changes, which can confirm hypogonadism and thus allows a tangible way to monitor testosterone replacement therapy. ${ }^{11}$ Literature highlights the need for better laboratory markers for patients with testosterone deficiency. 12

The objective of this study was to evaluate reproductive hormonal profile among three groups with varying degree of sexual performance satisfaction for erectile dysfunction with or without type-2 diabetes (T2DM). The secondary objective was to determine the association of inflammation, nephropathy, and glycation in different ED groups.

\section{METHODOLOGY}

This comparative cross-sectional study was conducted from January to December 2018 at the Pathology Department, PNS Hafeez Hospital, Islamabad, after approval from the Hospital's Ethical Review Committee. The target population included male subjects who had known diabetes for at least six months with age between 35 to 70 years. Subjects who had known history of hypertension, ischemic heart disease, or had any chronic or acute disease process like patients with type-1 diabetes, autoimmune disorder, cancer, recent medical or surgical emergency, or having some acute infections were excluded from the study on initial interview. Agematched male controls living a healthy life with no known disease were also included.

After a formal interview explaining the nature, requirements, and study consequences, subjects were requested to visit laboratory in exact medical fasting status. Sampling was carried out on the basis of nonprobability convenience methods. All included subjects were interviewed as per written questionnaire, followed by examination for blood pressure, any stigma of chronic disease, anthropometric measures by two medical specialists including one endocrinologist along with other medical officer. Both controls and diabetic subjects were evaluated in a similar manner.

Ten $\mathrm{ml}$ of blood was collected in gel vacutainer without preservatives, Na-Flouride and EDTA bottle for measurement of fasting plasma glucose, male reproductive hormones, quantitative CRP (qCRP), HbA1c. Urinary albumin creatinine ratio (UACR) was also calculated from spot urine. During further workup, few samples were lost due to hemolysis, sample quantity insufficiency and lipaemia for some parameters where patients did not re-visit for follow-up repeat testing concluding with a sample size of 121 subjects. Thus missing values for few parameters could not be included in the analysis.

Glycated hemoglobin (HbA1c), male sex hormones including luteinizing hormone (LH), follicle stimulating hormone ( $\mathrm{FSH}$ ), total testosterone, sex hormone binding globulin (SHBG) and prolactin were analysed by chemiluminescent microparticle immunoassay (CMIA) on
Architect iSystem. Abbot diagnostics fasting plasma glucose was analysed on Selctra-ProM (Random access clinical chemistry analyser) for measuring glucose by using GPO-PAP method. qCRP and UACR were measured by using Merck kits on Selectra-ProM.

Erectile dysfunction was assessed based upon a questionnaire where after explaining about sexual performance satisfaction, the study participants were asked to select one option as: Not satisfied, satisfied, or completely satisfied. Free androgen index, as a marker for bioavailable testosterone, was calculated by formula suggested by Wilke et al. as: Total testosterone/SHBG $\mathrm{x}$ 100.13 Underlying inflammation was assessed by qCRP as normal if levels of qCRP were $<5 \mathrm{mg} / \mathrm{L}$ or raised if levels were $>5 \mathrm{mg} / \mathrm{L}$. Nephropathy was evaluated by utilising urinary albumin creatinine ratio (UACR) and levels $<2.5 \mathrm{mg} / \mathrm{mmol}$ were considered normal while levels $>2.5 \mathrm{mg} / \mathrm{mmol}$ as predictive of nephropathy.

Data collected on Excel 2013 was moved to SPSSversion 16. Erectile dysfunction was evaluated using groups made as per patient described sexual performance satisfaction for age, anthropometric measures, biochemical and hormonal parameters by utilising oneway ANOVA followed by post-hoc Tukey's comparison. Following that general linear model (GLM) were utilised to see the impact of inflammation and nephropathy using $\mathrm{HbA} 1 \mathrm{C}$ and $\mathrm{FAl}$ as dependent variable with sexual performance satisfaction as fixed and qCRP and UACR as random variables. A $p$-value of $<0.05$ was considered as significant.

\section{RESULTS}

Mean age of the subjects was $48.94 \pm 6.85$ years. Overall, $36.7 \%(n=43), 29.4 \%(n=38)$ and $33.8 \%(n=40)$ showed complete erectile dysfunction, just satisfied, and completely satisfied based upon their interview-based sexual performance satisfaction evaluation regardless of presence or absence of type-2 diabetes mellitus. In terms of inflammation, $31.43 \%$ patients with T2DM had raised qCRP in comparison to $24.56 \%$ of age-matched controls, $p=0.257$. Nephropathy as measured by UACR was found in 10/56 age-matched controls in comparison to $38 / 69$ patients with T2DM $(p<0.001)$. Differences between groups formulated based upon interview-based sexual performance satisfaction for age, anthropometric measures, and certain hormonal parameters were assessed between the groups and shown in Table I. Figure 1 highlights that for any particular degree of erectile dysfunction reported, glycated hemoglobin demonstrated a higher trend from subject with less sexual satisfaction to individuals who were sexually satisfied with their performance. Moreover, inflammation as measured by qCRP always followed a rise with rising $\mathrm{HbA1c}$ levels, identifying inflammation as more related with worsening diabetes than with sexual performance satisfaction. 
FAl was evaluated among the three erectile dysfunction groups as demonstrated in Figure 2. FAl levels were higher among subjects who showed no erectile dysfunction than subjects with lesser or least satisfied groups. FAl levels tend to decline among all erectile dysfunction groups once inflammation marker (qCRP)

Table I: Difference between various groups based upon sexual performance satisfaction for age, anthropometric measures and certain hormonal parameters as measured by one-way ANOVA.

\begin{tabular}{|c|c|c|c|c|c|}
\hline Parameter & $\begin{array}{c}\text { Sexual performance } \\
\text { satisfaction }\end{array}$ & $\mathrm{N}(\%)$ & Mean & SD & Sig. (2-tailed) \\
\hline Age (years) & $\begin{array}{c}\text { Not satisfied } \\
\text { Just satisfied } \\
\text { Satisfied } \\
\text { Total }\end{array}$ & $\begin{array}{c}43(35.8 \%) \\
38(31.6 \%) \\
39(32.5 \%) \\
120\end{array}$ & $\begin{array}{l}49.49 \\
48.53 \\
48.67 \\
48.92 \\
\end{array}$ & $\begin{array}{l}6.16 \\
7.75 \\
6.78 \\
6.85 \\
\end{array}$ & 0.791 \\
\hline Waist to hip ratio (WHpR) & $\begin{array}{l}\text { Not satisfied } \\
\text { Just satisfied } \\
\text { Satisfied } \\
\text { Total }\end{array}$ & $\begin{array}{c}43(35.5 \%) \\
38(31.4 \%) \\
40(33.0 \%) \\
121\end{array}$ & $\begin{array}{l}0.56 \\
0.55 \\
0.56 \\
0.56\end{array}$ & $\begin{array}{l}0.070 \\
0.051 \\
0.070 \\
0.065 \\
\end{array}$ & 0.285 \\
\hline Body mass index (BMI) & $\begin{array}{c}\text { Not satisfied } \\
\text { Just satisfied } \\
\text { Satisfied } \\
\text { Total }\end{array}$ & $\begin{array}{c}43(35.5 \%) \\
38(31.4 \%) \\
40(33.0 \%) \\
121\end{array}$ & $\begin{array}{l}27.64 \\
26.67 \\
28.65 \\
27.67\end{array}$ & $\begin{array}{l}5.08 \\
4.18 \\
5.87 \\
5.12 \\
\end{array}$ & 0.234 \\
\hline Luteinizing hormone (LH) $\mathrm{mIU} / \mathrm{mL}$ & $\begin{array}{l}\text { Not satisfied } \\
\text { Just satisfied } \\
\text { Satisfied } \\
\text { Total }\end{array}$ & $\begin{array}{c}43(35.5 \%) \\
38(31.4 \%) \\
40(33.0 \%) \\
121\end{array}$ & $\begin{array}{l}4.81 \\
4.17 \\
4.94 \\
4.65\end{array}$ & $\begin{array}{l}3.98 \\
2.11 \\
3.07 \\
3.17 \\
\end{array}$ & 0.522 \\
\hline Follicle stimulating hormone (FSH) $\mathrm{mlU} / \mathrm{mL}$ & $\begin{array}{c}\text { Not satisfied } \\
\text { Just satisfied } \\
\text { Satisfied } \\
\text { Total }\end{array}$ & $\begin{array}{c}43(35.5 \%) \\
38(31.4 \%) \\
40(33.0 \%) \\
121\end{array}$ & $\begin{array}{l}6.38 \\
5.36 \\
6.59 \\
6.13 \\
\end{array}$ & $\begin{array}{l}6.68 \\
2.83 \\
4.81 \\
5.17 \\
\end{array}$ & 0.538 \\
\hline Prolactin (mlU/L) & $\begin{array}{l}\text { Not satisfied } \\
\text { Just satisfied } \\
\text { Satisfied } \\
\text { Total }\end{array}$ & $\begin{array}{c}43(35.5 \%) \\
38(31.4 \%) \\
40(33.0 \%) \\
120\end{array}$ & $\begin{array}{l}219 \\
185 \\
204 \\
203 \\
\end{array}$ & $\begin{array}{c}172.81 \\
79.30 \\
163.90 \\
203.93\end{array}$ & 0.569 \\
\hline HbA1c (\%) & $\begin{array}{l}\text { Not satisfied } \\
\text { Just satisfied } \\
\text { Satisfied } \\
\text { Total }\end{array}$ & $\begin{array}{c}43(35.8 \%) \\
38(31.6 \%) \\
39(32.5 \%) \\
120\end{array}$ & $\begin{array}{l}7.88 \\
6.70 \\
7.01 \\
7.21\end{array}$ & $\begin{array}{l}2.60 \\
2.09 \\
2.00 \\
2.29\end{array}$ & 0.056 \\
\hline
\end{tabular}

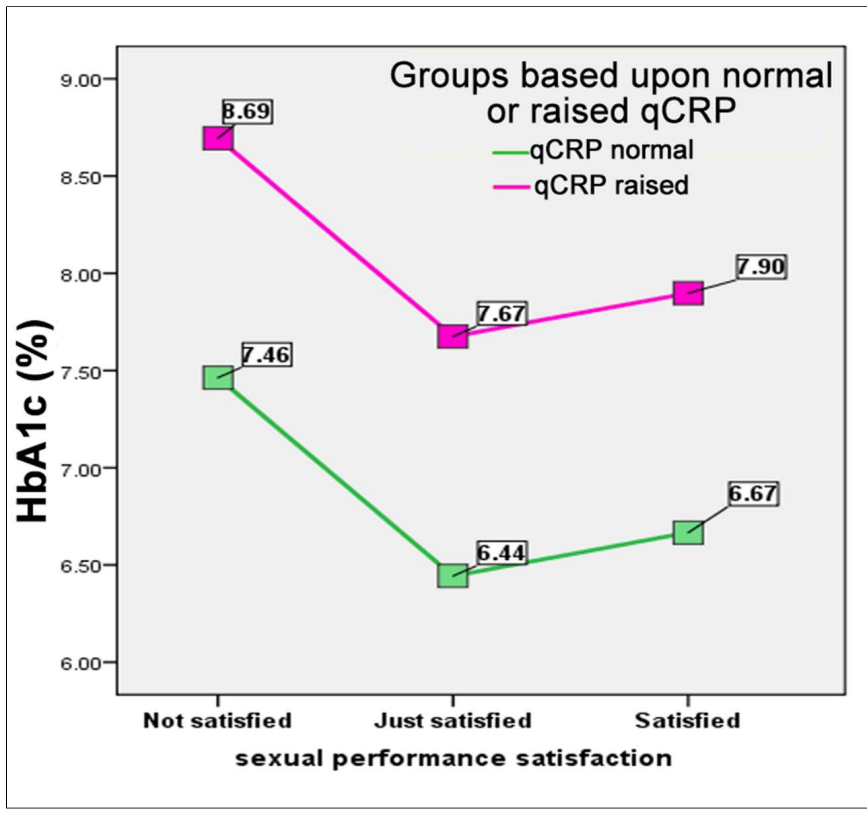

Figure 1: General linear model (GLM) using $\mathrm{HbA} 1 \mathrm{c}$ as dependent variable with sexual performance satisfaction as fixed and qCRP as random variables. (Model significance: $p<0.052, n=119$ ).



Figure 2: General linear model (GLM) using free androgen index (FAI) as dependent variable with sexual performance satisfaction as fixed and qCRP as random variables. (Model significance: $p<0.046$ ), $n=120$ ). 


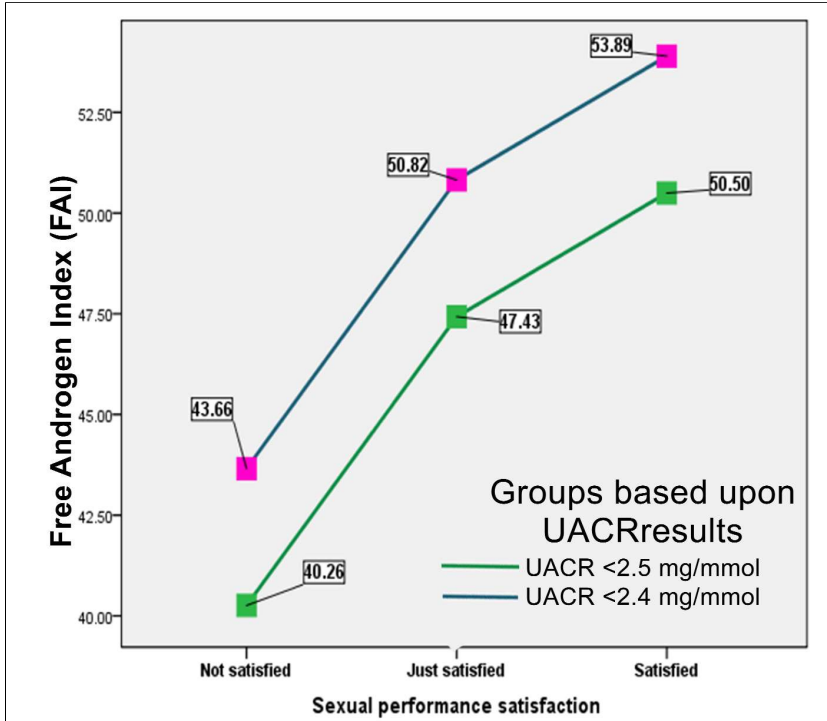

Figure 3: General linear model (GLM) using free androgen index (FAl) as dependent variable with sexual performance satisfaction as fixed and UACR as random variables. (Model significance: $p<0.023, n=119$ ).

was high. Similarly, UACR demonstrated a similar worsening pattern where the levels were higher among all erectile dysfunction groups as shown in Figure 3.

\section{DISCUSSION}

This cross-sectional study showed that glycation index, i.e. HbA1c levels, was higher among subjects with poor sexual performance satisfaction group in comparison to subjects who gave history of optimal sexual performance satisfaction. This finding identifies an association between glycemia and hypogonadism. Moreover, all these sexual performance satisfaction-based groups were found to have higher inflammation along $\mathrm{HbA1c}$ levels. This observation along with association of $\mathrm{FAl}$ and sexual performance satisfaction (ED groups) also indicated that inflammation levels rise from satisfied subjects to patient with complete erectile dysfunction. Moreover, subjects within the three ED groups demonstrated increasing nephropathy as evaluated by UACR along with FAI. These results suggest that subjects' risk of androgen deficiency and ED were more associated with raised $\mathrm{HbA1c}$, inflammation, and nephropathy. This finding was prevalent among both controls and diabetic subjects with slightly higher frequency in subjects with poor glycemic controls as depicted by higher HbA1c levels.

Generally, literature suggests a pattern suggesting hypogonadotropic hypogonadism in male subjects with T2DM as what appeared in our findings. ${ }^{14}$ However, the literature review also highlights some contrasting data on the patterns of about repoductive hormones in T2DM patients with hypogonadism. Elsaied et al. have not found any direct link between testosterone levels and ED, but were able to show an association of testosterone with insulin resistance markers. ${ }^{15}$ Similarly, a Chinese study did not demonstrate an association between testosterone and T2DM, but showed low SHBG to be associated with T2DM risk. ${ }^{16}$ Brand JS et al. in their reviewed data on metabolic syndrome and reproductive hormones could only found testosterone deficiency in the metabolic syndrome but only after adjustment of multiple factors including BMI, serum triglycerides, and hyperglycemia. ${ }^{17}$ In contrast to the above, Gyawali et al. identified low total testosterone but not SHBG in patients with T2DM in an off-shoot study from 'The men androgen inflammation lifestyle and stress study' (MAILES). ${ }^{18}$ These studies highlight a lot of contrasting evidence in literature and possible explanation linking the effects of glucotoxicity associated with T2DM and male reproductive hormones. Firstly, the authors believe that hormonal differences between multiple studies may be related to recently identified multiple molecular differences within the term T2DM as not all patients with T2DM have been shown to present study with same micro- and macro-vessel complications or a need arises to segregate the broader label of T2DM into subtypes as highlighted in few studies. ${ }^{19}$ Erectile dysfunction and T2DM are disease categories where a lot many confounders can act as contributors as highlighted in the study by Brand JS et al., so the differences between the researches could be attributed to related factors like obesity. ${ }^{17}$ Furthermore, total testosterone, and even free testosterone in isolation, may not show complete evidence about ED, and therefore, the combined factorial aspects of SHBG with testosterone may be considered for utilisation. The authors' interpretation is that both subjects and controls once having associated inflammation or complications of diabetes like higher glycation or nephropathy are more associated with less sexual performance satisfaction, so studies dealing only with newly diagnosed cases of diabetes may not have yet progressed to the stage of micro-vascular complications of T2DM. So, this could be one more factor in the differences in data as highlighted above. Though question have been raised about the FAI utility in males, Guay et al. have considered the variance to increase while using calculated methods to assess androgen levels. ${ }^{20}$ However, keeping in view the data contrast from aforementioned shared studies on SHBG and testosterone, we feel there combined use may be more fruitful in assessing hypogonadism as suggested by some researchers. ${ }^{21,22}$

Proof of evidence for our findings can be viewed from trials using testosterone replacement therapy (TRT), where few researchers apart from highlighting cardiovascular concerns have shown benefits of managing deficiency. ${ }^{23}$ However, data on TRT currently favours the use of TRT with both beneficial effects in terms of survival benefits in T2DM along with improvement in erectile dysfunction and testosterone levels. ${ }^{24,25}$

Certain limitations to our study need to be acknowledged. Firstly, this was a comparative cross-sectional study as 
what we could do within our hospital setting within the available resource, which may suffer the possibility of type-2 statistical error. Secondly, being an observational study, the study has been able to highlight a trend of male patients suffering from hypogonadism within T2DM group and otherwise; so two epidemiological/ RCTs must follow to evaluate the link between male hypogonadism and reproductive hormones to establish cut-offs for FAI and other parameters. Thirdly, there should be a multivariate analysis to analyse the effect size of confounders in contributing towards both biochemical and clinical hypogonadism.

Though the article has some limitations as highlighted above, T2DM-associated complication evaluation should routinely include an assessment of hypogonadism in males, especially in our set-ups. Secondly, the research was able to highlight patient's defined sexual satisfaction is associated with possibility of not only hypogonadism, but also these subjects suffer from higher degree of inflammation, nephropathy and hemoglobin glycation. Thus, male hypogonadism must be viewed with regards to both reproductive function, but also its probable association with cardiovascular and renal risks due to inflammation, nephropathy and higher rates of glycation. ${ }^{3}$

\section{CONCLUSION}

Sexual performance satisfaction and FAI declines with rise in glycated hemoglobin. Moreover, the subjects having underlying complications like nephropathy or higher inflammation were found to have lower FAI and increased frequency of ED both in controls and T2DM.

\section{ETHICAL APPROVAL:}

The study was formally approved by the Hospital's Ethical Review Committee prior to the start of the study.

\section{PATIENTS' CONSENT:}

All participants signed written informed consent before study, after explanation about use of data for research.

\section{CONFLICT OF INTEREST:}

Authors declared no conflict of interest.

\section{AUTHORS' CONTRIBUTION:}

SHK: Study conceptualization, Lab analytic analysis, manuscript preparation.

RA: Sampling, history and clinical examination, data analysis, writing manuscript.

ZAA: History and sampling, SPSS data analysis, manuscript writing.

NF: Patient selection and initial clinical evaluation, results analysis, manuscript writing.

AASG: Patient history and clinical evaluation, contribution in manuscript writing.

MT: Sample selection, medical writing, and overall study coordination.

\section{REFERENCES}

1. Lopez-de-Andres A, Jimenez-Garcia R, Hernandez-Barrera V, Jimenez-Trujillo I, Gallardo-Pino C, de Miguel AG, CarrascoGarrido. National trends over one decade in hospitalization for acute myocardial infarction among Spanish adults with type 2 diabetes: Cumulative incidence, outcomes and use of percutaneous coronary intervention. PLoS One 2014; 9: e85697.

2. Agrawal RP, Sharma P, Pal M, Kochar A, Kochar DK. Magnitude of dyslipedemia and its association with micro and vascular complications in type 2 diabetes: A hospital based study from Bikaner (Northwest India). Diabetes Res Clin Pract 2006; 73:211-4.

3. Corona G, Giorda CB, Cucinotta D, Guida P, Nada E. Gruppo di studio SUBITO-DE. Sexual dysfunction at the onset of type 2 diabetes: The interplay of depression, hormonal and cardiovascular factors. J Sex Med 2014; 11:2065-73.

4. Livingston M, Kalansooriya A, Hartland AJ, Ramachandran S, Heald A. Serum testosterone levels in male hypogonadism: Why and when to check - A review. Int J Clin Pract 2017; 71: e12995.

5. Staerman F, Léon P. Andropause (androgen deficiency of the aging male): Diagnosis and management. Minerva Med 2012; 103:333-42.

6. Dandona P, Dhindsa S. Update: Hypogonadotropic hypogonadism in type 2 diabetes and obesity. $J$ Clin Endocrinol Metab 2011; 96:2643-51.

7. Kiskac M, Zorlu M, Cakisrca M, Büyükaydin B, Karatoprak C, Yavuz E. Frequency and determinants of erectile dysfunction in Turkish diabetic men. Niger J Clin Pract 2015; 18:209-12.

8. Lu CC, Jiann BP, Sun CC, Lam HC, Chu CH, Lee JK. Association of glycemic control with risk of erectile dysfunction in men with type 2 diabetes. J Sex Med 2009; 6:1719-28.

11. Cho NH, Ahn CW, Park JY, Ahn TY, Lee HW, Park TS, et al. Prevalence of erectile dysfunction in Korean men with Type 2 diabetes mellitus. Diabet Med 2006; 23:198-203.

12. Ahmed I, Aamir Au, Anwar E, Ali SS, Ali A, Ali A. Erectile dysfunction and type 2 diabetes mellitus in northern Pakistan. J Pak Med Assoc 2013; 63:1486-90.

11. Blick G. Optimal diagnostic measures and thresholds for hypogonadism in men with HIVIAIDS: Comparison between 2 transdermal testosterone replacement therapy gels. Postgrad Med 2013; 125:30-9.

12. Morales A. Testosterone deficiency syndrome: An overview with emphasis on the diagnostic conundrum. Clin Biochem 2014; 47:960-6.

13. Wilke TJ, Utley DJ. Total testosterone, free-androgen index, calculated free testosterone, and free testosterone by analog RIA compared in hirsute women and in otherwise-normal women with altered binding of sex-hormone-binding globulin. Clin Chem 1987; 33:1372-5.

14. Dhindsa S, Ghanim H, Batra M, Dandona P. Hypogonadotropic hypogonadism in men with diabesity. Diabetes Care 2018; 41: 1516-25.

15. Elsaied MA, Masallat D, Abdel-Hamid IA. Correlation of adiponectin with testosterone in patients with and without type 2 diabetes and erectile dysfunction. Am J Mens Health 2018; 19:1557988318807049. 
16. Hu J, Zhang A, Yang S, Wang Y, Goswami R, Zhou H, et al. Combined effects of sex hormone-binding globulin and sex hormones on risk of incident type 2 diabetes. J Diabetes 2016; 8:508-15.

17. Brand JS, van der Tweel I, Grobbee DE, Emmelot-Vonk MH, van der Schouw YT. Testosterone, sex hormone-binding globulin and the metabolic syndrome: A systematic review and meta-analysis of observational studies. Int $\mathrm{J}$ Epidemiol 2011; 40:189-207.

18. Gyawali P, Martin SA, Heilbronn LK, Vincent AD, Taylor AW, Adams RJT, et al. The role of sex hormone-binding globulin (SHBG), testosterone, and other sex steroids, on the development of type 2 diabetes in a cohort of community-dwelling middle-aged to elderly men. Acta Diabetol 2018; 55:861-872.

19. Udler MS, Kim J, von Grotthuss M, Bonàs-Guarch S, Cole JB, Chiou J, et al. Type 2 diabetes genetic loci informed by multitrait associations point to disease mechanisms and subtypes: A soft clustering analysis. PLoS Med 2018; 15:e1002654.

20. Guay AT, Traish AM, Hislop-Chestnut DT, Doros G, Gawoski JM. Are there variances of calculated free testosterone attributed to variations in albumin and sex hormone-binding globulin concentrations in men? Endocr Pract 2013; 19:236-42.

21. Du JP, Li FP, Zheng XB, Lan BY, Lin DZ, Li Y, et al. Diagnostic criteria for late-onset hypogonadism: A preliminary study. Zhonghua Nan Ke Xue 2016; 22:979-83.

22. Sartorius G, Ly LP, Sikaris K, McLachlan R, Handelsman DJ. Predictive accuracy and sources of variability in calculated free testosterone estimates. Ann Clin Biochem 2009; 46:137-43.

23. Corona G, Rastrelli G, Maseroli E, Fralassi N, Sforza A, Forti G, et al. Low testosterone syndrome protects subjects with high cardiovascular risk burden from major adverse cardiovascular events. Andrology 2014; 2:741-7.

24. Muraleedharan V, Marsh H, Kapoor D, Channer KS, Jones TH. Testosterone deficiency is associated with increased risk of mortality and testosterone replacement improves survival in men with type 2 diabetes. Eur J Endocrinol 2013; 169:725-33.

25. Efesoy O, Çayan S, Akbay E. The effect of testosterone replacement therapy on penile hemodynamics in hypogonadal men with erectile dysfunction, having veno-occlusive dysfunction. Am J Mens Health 2018; 12:634-8.

$\cdots \cdot \hat{r} \cdot \ldots$ 